\title{
ADAPTIVE DYNAMICS IN LOGISTIC BRANCHING POPULATIONS
}

\author{
NICOLAS CHAMPAGNAT \\ TOSCA team \\ INRIA Sophia Antipolis-Méditerrannée \\ 2004 Route des Lucioles, BP 93, 06902 Sophia Antipolis, France \\ E-mail: Nicolas.Champagnat@sophia.inria.fr
}

AMAURY LAMBERT

Unit of Mathematical Evolutionary Biology

CNRS UMR 7625 Laboratoire d'Écologie et Évolution

École Normale Supérieure

46 rue d'Ulm, 75230 Paris Cedex 05, France

E-mail: amaury.lambert@ens.fr

and

CNRS UMR 7625 Laboratoire d'Écologie et Évolution

UPMC Univ. Paris 06

7 quai Saint Bernard, 75252 Paris Cedex 05, France

\begin{abstract}
The biological theory of adaptive dynamics proposes a description of the long-time evolution of an asexual population, based on the assumptions of large population, rare mutations and small mutation steps. Under these assumptions, the evolution of a quantitative dominant trait in an isolated population is described by a deterministic differential equation called 'canonical equation of adaptive dynamics'. In this work, in order to include the effect of genetic drift in this model, we consider instead finite, randomly fluctuating populations and weak selection.

We consider a trait-structured population subject to mutation, birth and competition of logistic type, where the number of coexisting types may fluctuate. Applying a limit of rare mutations to this population while keeping the population size finite leads to a jump process, the so-called 'trait substitution sequence', where evolution proceeds by successive invasions and fixa-
\end{abstract}

2000 Mathematics Subject Classification: Primary 92D15; Secondary 60K35, 60J85, 60J70, 92D10, 60J75, 92D25, 92D40, 60J80, 60J25.

Key words and phrases: logistic branching process, multitype birth death competition process, density-dependence, fixation probability, genetic drift, weak selection, adaptive dynamics, invasion fitness, timescale separation, trait substitution sequence, diffusion approximation.

The paper is in final form and no version of it will be published elsewhere. 
tions of mutant types. The probability of fixation of a mutant is interpreted as a fitness landscape that depends on the current state of the population. Rescaling mutation steps (weak selection) then yields a diffusion on the trait space christened 'canonical diffusion of adaptive dynamics', in which genetic drift (diffusive term) is combined with directional selection (deterministic term) driven by the fitness gradient.

Finally, in order to compute the coefficients of this diffusion, we seek explicit first-order formulae for the probability of fixation of a nearly neutral mutant appearing in a resident population. The first-order term is a linear combination of products of functions of the initial mutant frequency times 'invasibility coefficients' associated with fertility, defence, aggressiveness and isolation, which measure the robustness (stability with respect to selective strengths) of the resident type. Some numerical results on the canonical diffusion are also given.

1. Introduction. The recent biological theory of adaptive dynamics [6,9] proposes a description of the long term evolution of an asexual population by putting emphasis on the ecological interactions between individuals, in contrast with classical population genetics models which focus on the genetic structure of the population. The basic models are individual-based models in which the population dynamics is precisely described and includes birth, death, competition and mutation. The basic idea of the theory of adaptive dynamics is to try to get insights about the interplay between ecology and evolution by studying the invasion of a single mutant type appearing in a simplified (monotype stable) resident population. The evolution of the population can then be described as a sequence of mutant invasions in the population. If the resident type goes extinct when the mutant type invades (we say that the mutant type becomes fixed), the evolution is described by the so-called 'trait substitution sequence' (TSS) [10]. This approach has revealed powerful to predict the qualitative behaviour of complex evolutionary dynamics. In particular, it allows one to determine the (local) direction of evolution in the space of phenotypic traits (or simply traits) from the individual ecological parameters, and to predict and explain the phenomenon of evolutionary branching [10], where a population, initially composed of individuals with traits concentrated around a single trait value, divides into two (or more) subpopulations concentrated around distinct trait values that stably coexist because of their ecological interactions. The description of this phenomenon is an important achievement of this theory, as well as the 'canonical equation of adaptive dynamics' [3], which describes the evolution of the dominant trait of the population as a deterministic "hill-climbing" process on a fitness landscape which depends on the current state of the population (see (1) below).

More formally, as soon as eternal coexistence of two or more types is not permitted by the model, the evolution can be described by the sequences $\left(T_{n}\right)_{n}$ and $\left(V_{n}\right)_{n}$, where $T_{n}$ is the $n$-th time where the population becomes monomorphic (i.e. composed of only one type) and $V_{n}$ is the surviving type at time $T_{n}$. The sequence $\left(V_{n}\right)$ is the above-mentionned TSS. It is possible to prove the convergence of an individual-based model to the TSS under two biologically motivated assumptions [10,1]. First, the assumption of rare mutations guarantees that, in the timescale of mutations, the widths of time intervals during which the population is polymorphic vanish, so that there is only one type surviving at any time $t$. To prevent the population from rapidly becoming 
extinct in the new timescale, one also has to rescale population sizes, thereby making the assumption of large populations.

Subsequently, the TSS is a Markov jump process on the trait space whose semigroup is shown [1] to depend on the invasion fitnesses ([9]) $f(x, y), x, y \in \mathcal{X}$, where $f(x, y)$ is defined as the expected growth rate of a single individual of type $y$ - the mutant entering a monomorphic population of type $x$ 'at equilibrium' - the residents. Note that this fitness is not given a priori, but derived from the microscopic model of individual interactions. Because of the assumption of large population, the sign of this fitness determines the possibility of invasion of a mutant type: if $f(x, y)<0$, the mutant type $y$ cannot invade a resident population of type $x$. Thus, evolution proceeds by successive invasions of (only) advantageous mutant types replacing the resident one.

The 'canonical equation of adaptive dynamics' [3], which describes the evolution of a one-dimensional trait $x$ as the solution of the following ODE, is obtained from the TSS in the limit of small mutations:

$$
\frac{d x}{d t}=\frac{1}{2} \sigma(x)^{2} \mu(x) \bar{n}(x) \frac{\partial}{\partial y} f(x, x),
$$

where $\sigma(x)^{2}$ stands for the (rescaled) variance of the mutation step law, $\bar{n}(x)$ for the equilibrium size of a pure $x$-type population, and $f(x, y)$ for the invasion fitness mentioned above. Note how only advantageous types get fixed (the trait follows the fitness gradient) and how the fitness landscape $y \mapsto f(x, y)$ depends on the current state $x$ of the population.

However, it is well-known that slightly deleterious types can be fixed by chance in finite populations. This phenomenon is known under the name of genetic drift. Depending on the strength of genetic drift, selection is said to be strong (genetic drift has negligible effects) or weak. In the large population asymptotic from which the TSS of adaptive dynamics is derived, genetic drift has negligible impact compared to the action of selection. Therefore, the fixation of slightly deleterious types cannot be observed. Our goal here is to include genetic drift in the adaptive dynamics models by considering finite populations under weak selection. We continue using the bottom-up approach of adaptive dynamics; that is, model (macroscopic) evolution from (microscopic) populations. In particular, we allow the population sizes to fluctuate randomly through time and we aim to reconstruct a fitness function from the microscopic parameters.

After the description of the model (Section 2), we derive a new TSS in the limit of rare mutations (Section 3), from which a limit of small mutations gives what we call the 'canonical diffusion of adaptive dynamics' (Section 4). The coefficients of this diffusion involve the first-order derivatives of the fixation probabilities, which are computed in Section 5 as a linear combination of four fundamental components associated to fertility, defence, aggressiveness and isolation. New numerical results on the robustness of the population with respect to these fundamental components are also given, as well as some consequences on the canonical diffusion of adaptive dynamics in large populations.

2. The microscopic model. We will restrict here to logistic interaction. More general models are considered in [2]. 
A monotype (binary) logistic branching process (LBP, see [7]) with dynamical parameters $(b, c)$ is a Markov chain in continuous time $\left(X_{t} ; t \geq 0\right)$ with nonnegative integer values and transition rates

$$
q_{i j}= \begin{cases}b i & \text { if } j=i+1 \\ c i(i-1) & \text { if } j=i-1 \\ -i(b+c(i-1)) & \text { if } j=i \\ 0 & \text { otherwise. }\end{cases}
$$

The non-linear term $c i(i-1)$ describes competition mortality due to random encounters between individuals. Other terms correspond to independent birth events with constant individual rates. This Markov chain is positive-recurrent and converges in distribution to a r.v. $\xi$, where $\xi$ is a Poisson variable of parameter $\theta:=b / c$ conditioned on being nonzero

$$
\mathbb{P}(\xi=i)=\frac{e^{-\theta}}{1-e^{-\theta}}, \frac{\theta^{i}}{i !}, \quad i \geq 1 .
$$

We consider a multitype asexual birth and death process with mutation, generalizing this LBP. At any time $t$, the population is composed of a finite number $N(t)$ of individuals characterized by their phenotypic traits $x_{1}(t), \ldots, x_{N(t)}(t)$ belonging to a given trait space $\mathcal{X}$, assumed to be a closed subset of $\mathbb{R}^{k}$. The population state at time $t$ is represented by the counting measure on $\mathcal{X}$

$$
\nu_{t}=\sum_{i=1}^{N(t)} \delta_{x_{i}(t)} .
$$

The population dynamics is governed by the following parameters.

- $b(x)$ is the rate of birth from an individual of type $x$. The function $b$ is assumed to be $\mathcal{C}_{b}^{2}$.

- $c(x, y)$ is the rate of death of an individual of type $x$ due to the competition with another individual of type $y$. Therefore, the total death rate of an individual of type $x$ in a population $\nu$ may be written as $\int c(x, y)\left(\nu(d y)-\delta_{x}(d y)\right)$. In this expression, the Dirac mass at $x$ substracted to the measure $\nu$ means that the individual does not compete with himself. The function $c$ is assumed to be $\mathcal{C}_{b}^{2}$ and bounded away from 0 on $\mathcal{X}^{2}$.

- $\gamma \mu(x)$ is the probability that a birth from an individual with trait $x$ produces a mutant individual, where $\mu(x) \in[0,1]$ and where $\gamma \in(0,1]$ is a parameter scaling the frequence of mutations. When there is no mutation, the new individual inherits the trait of its progenitor. In Section 3, we will be interested in the limit of rare mutations $(\gamma \rightarrow 0)$.

- $M(x, d h)$ is the law of the trait difference $h=y-x$ between a mutant individual with trait $y$ and the trait $x$ of his progenitor. We assume that $M(x, d h)$ has 0 expectation (no mutation bias), i.e. $\int h M(x, d h)=0$, and has a density on $\mathbb{R}^{k}$ which is uniformly bounded in $x \in \mathcal{X}$ by some function $\bar{M}(h)$ with finite third-order moment.

We will denote the dependence of $\nu_{t}$ on the parameter $\gamma$ with the notation $\nu_{t}^{\gamma}$. Observe that such a population cannot become extinct because the death rate is 0 when there is only one individual in the population. Since we want to apply a limit of rare mutations 
while keeping the population size finite, this is necessary to prevent the population to become extinct before any mutations occur.

Let $\xi(x)$ be a random variable whose law is the stationary distribution of a pure $x$-type population with no mutation $(\mu \equiv 0)$. This law is given by $(2)$ where $\theta$ is replaced by $\theta(x):=b(x) / c(x, x)$.

The last notation needed concerns a population with initially only two types $x$ and $y$ and with no mutation. Then $\nu_{t}=X_{t} \delta_{x}+Y_{t} \delta_{y}$, where $\left(X_{t}, Y_{t}: t \geq 0\right)$ is a bivariate Markov chain. For this Markov chain, $\mathbb{P}(T<\infty)=1$, where $T$ is the first time where either $X_{t}$ or $Y_{t}$ reach 0 . We call fixation (of the mutant $y$ ) the event $\left\{X_{T}=0\right\}$. The probability of fixation will be denoted by $u_{n, m}(x, y)$

$$
u_{n, m}(x, y):=\mathbb{P}\left(X_{T}=0 \mid X_{0}=n, Y_{0}=m\right) .
$$

3. The trait substitution sequence in finite populations. In this section, we apply the limit of rare mutations $(\gamma \rightarrow 0)$ to the process $\nu^{\gamma}$, in order to describe the evolution of the population as a TSS in finite population. This limit requires to rescale time properly, as $t / \gamma$, to describe the evolution on the mutation timescale.

Theorem 1. Fix $x \in \mathcal{X}$. Assume that $\nu_{0}^{\gamma}=N_{0}^{\gamma} \delta_{x}$ where $\sup _{\gamma \in(0,1)} \mathbb{E}\left(\left(N_{0}^{\gamma}\right)^{p}\right)<\infty$ for some $p>1$. Then, for any $0<t_{1}<\ldots<t_{n}$, the $n$-tuple $\left(\nu_{t_{1} / \gamma}^{\gamma}, \ldots, \nu_{t_{n} / \gamma}^{\gamma}\right)$ converges in law for the weak topology to $\left(N_{t_{1}} \delta_{S_{t_{1}}}, \ldots, N_{t_{n}} \delta_{S_{t_{n}}}\right)$ where

(1) $\left(S_{t} ; t \geq 0\right)$ is a Markov jump process on $\mathcal{X}$ with initial value $S_{0}=x$ and whose jumping rates $q(x, d h)$ from $x$ to $x+h$ are given by

$$
q(x, d h)=\beta(x) \chi(x, x+h) M(x, d h),
$$

where $\beta(x)=\mu(x) b(x) \mathbb{E}(\xi(x))=\mu(x) b(x) \theta(x) /\left(1-e^{-\theta(x)}\right)$ and

$$
\chi(x, y)=\sum_{n \geq 1} \frac{n \mathbb{P}(\xi(x)=n)}{\mathbb{E}(\xi(x))} u_{n, 1}(x, y)=\sum_{n \geq 1} e^{-\theta(x)} \frac{\theta(x)^{n-1}}{(n-1) !} u_{n, 1}(x, y) .
$$

(2) Conditional on $\left(S_{t_{1}}, \ldots, S_{t_{n}}\right)=\left(x_{1}, \ldots, x_{n}\right)$, the $N_{t_{i}}$ are independent and respectively distributed as $\xi\left(x_{i}\right)$.

Therefore, in the limit of rare mutations, on the mutation timescale, the population is always monomorphic and the dominant trait of the population evolves as a jump process over the trait space, where a jump corresponds to the appearance and fixation of a mutant type. Moreover, at any time, the population size is stationary (i.e. has the stationary distribution corresponding to the dominant trait of the population). The fixation rate of a mutant is governed by the function $\chi(x, y)$, which is therefore the random analogue of the traditional invasion fitness [9], defined as the probability of invasion of a mutant type $y$ in a resident population of type $x$ at equilibrium. Observe that, as usual in adaptive dynamics, the fitness landscape depends on the current state of the population. Moreover, in contrast with the classical TSS [10], from a given monomorphic resident population, any mutant trait has a positive probability to invade (genetic drift). Therefore, evolution is possible in any direction of the trait space. However, selection still acts in a preferential direction, as will appear in the next section. 
We refer to [2] for the proof of this result. However, this convergence is natural in view of the following interpretation of each parameter. The quantity $\beta(x)$ can be seen as the mean mutant production rate of a stationary $x$-type population (i.e. with size $\xi(x))$, and $\chi(x, y)$ is the probability of fixation of a single $y$-type mutant entering a pure $x$-type population with size-biased stationary size. The size bias comes from the fact that the mutant appears at a birth time in the stationary population (since the birth rate is proportional to the population size, the population size after a birth event in the stationary population is given by the size-biaised stationary population size).

4. The canonical diffusion of adaptive dynamics. Let us assume for simplicity that $\mathcal{X}=\mathbb{R}^{k}$. Let $\sigma(x)$ be the square root matrix of the covariance matrix of $M(x, \cdot)$. We also need to assume that the matrix $\sigma(x)$ is a Lipschitz function of $x$.

In order to obtain the equivalent of the canonical equation of adaptive dynamics in a finite population, we want to apply a limit of small mutation steps (weak selection) to the TSS $S$. To this aim, we introduce a parameter $\epsilon>0$ and replace the mutation kernels $M(x, \cdot)$ by their image by the application $h \mapsto \epsilon h$. Time also has to be rescaled in order to obtain a non-degenerate limit. The correct time scaling is $1 / \epsilon^{2}$, which leads to the following generator for the rescaled $\operatorname{TSS}\left(S_{t}^{\epsilon} ; t \geq 0\right)$

$$
A_{\epsilon} \varphi(x)=\frac{1}{\epsilon^{2}} \int_{\mathbb{R}^{k}}(\varphi(x+\epsilon h)-\varphi(x)) \beta(x) \chi(x, x+\epsilon h) M(x, d h) .
$$

Using the assumption that the mutation kernels $M(x, \cdot)$ have 0 expectation, it is elementary to compute the limit of this expression as $\epsilon \rightarrow 0$ (for sufficiently regular $\varphi$ ). This limit, which takes the form of a diffusion generator, explains the following result (its full proof can be found in [2]).

THEOREM 2. If the family $\left(S_{0}^{\epsilon}\right)_{\epsilon>0}$ has bounded first-order moments and converges in law as $\epsilon \rightarrow 0$ to a random variable $Z_{0}$, then the process $S^{\epsilon}$ with initial state $S_{0}^{\epsilon}$ converges in law for the Skorohod topology on $\mathbb{D}\left(\mathbb{R}_{+}, \mathbb{R}^{k}\right)$ to the diffusion process $\left(Z_{t} ; t \geq 0\right)$ with initial state $Z_{0}$, the unique solution to the stochastic differential equation

$$
d Z_{t}=\beta\left(Z_{t}\right) \sigma^{2}\left(Z_{t}\right) \cdot \nabla_{2} \chi\left(Z_{t}, Z_{t}\right) d t+\sqrt{\beta\left(Z_{t}\right) \chi\left(Z_{t}, Z_{t}\right)} \sigma\left(Z_{t}\right) \cdot d B_{t}
$$

where $\nabla_{2} \chi$ denotes the gradient w.r.t. the second variable $y$ of $\chi(x, y)$ and $B$ is a standard $k$-dimensional Brownian motion.

This result gives the equivalent form of the canonical equation of adaptive dynamics (1) when the population is finite. It is no longer a deterministic ODE, but a diffusion process, in which the genetic drift remains present (in the form of a stochastic diffusion term), as a consequence of the population finiteness and of the limit of weak selection. The deterministic drift part of (4) is very similar to the standard canonical equation of adaptive dynamics (1), and involves in particular the gradient of the fitness function $\chi$. The process (4) provides a diffusion model describing the evolution of the dominant trait value in a population $[8,5]$, grounded on a precise microscopic density-dependent modelling of the population dynamics. It also gives the precise balance between directional selection and genetic drift as a function of the individual's dynamical parameters. 
5. Fixation probability near neutrality. The SDE (4) involves the fixation probability $\chi(x, x)$ and the fitness gradient with respect to the second variable $\nabla_{2} \chi(x, x)$. In this section, we explain how these quantities can be explicitly computed.

We need to compute the derivatives of the fixation probabilities $u_{n, m}(x, y)$ when $y$ is close to $x$. Recall that the law of the two-type LBP without mutation $(X, Y)$ used to define $u_{n, m}$ in the end of Section 2 is characterized by the birth vector $B$ and the competition matrix $C$

$$
B=\left(\begin{array}{l}
b(x) \\
b(y)
\end{array}\right), \quad C=\left(\begin{array}{ll}
c(x, x) & c(x, y) \\
c(y, x) & c(y, y)
\end{array}\right) .
$$

We will say that the mutant is neutral if all individuals are exchangeable, i.e. when $b(y)=b(x)$ and $c(x, y)=c(y, x)=c(y, y)=c(x, x)$ (this holds in particular when $y=x)$. As will appear below, using the notation $(b, c):=(b(x), c(x, x))$, it is natural to focus on deviations from the neutral case expressed as

$$
B=b \mathbf{1}+\left(\begin{array}{c}
0 \\
\lambda
\end{array}\right), \quad C=c \mathbf{1}-\left(\begin{array}{cc}
0 & 0 \\
\delta & \delta
\end{array}\right)+\left(\begin{array}{cc}
0 & \alpha \\
0 & \alpha
\end{array}\right)-\left(\begin{array}{ll}
0 & \varepsilon \\
\varepsilon & 0
\end{array}\right) .
$$

In words, deviations from the neutral case are a linear combination of four fundamental selection coefficients $\lambda, \delta, \alpha, \varepsilon$, that are chosen to be positive when they confer an advantage to the mutant. It is convenient to assess these deviations to the neutral case in terms of

1. fertility $(\lambda)$ : positive $\lambda$ means increased mutant birth rate;

2. defence capacity $(\delta)$ : positive $\delta$ means reduced competition sensitivity of mutant individuals w.r.t. the total population size;

3. aggressiveness $(\alpha)$ : positive $\alpha$ means raised competition pressure exerted from any mutant individual onto the rest of the population;

4. isolation $(\varepsilon)$ : positive $\varepsilon$ means lighter cross-competition between the two different types, that would lead, if harsher, to a greater probability of exclusion of the less abundant one.

Under neutrality, an elementary martingale argument shows that the fixation probability $u_{n, m}$, further denoted $u$, equals the initial mutant frequency $p:=m /(m+n)$. This implies in particular that

$$
\chi(x, x)=\frac{e^{-\theta(x)}-1+\theta(x)}{\theta(x)^{2}} .
$$

The following theorem unveils the dependence of $u$ upon $\lambda, \delta, \alpha$,, when they slightly deviate from 0 , and explains why these four selection coefficients provide a natural basis to decompose the gradient of the fixation probability.

THEOREM 3. As a function of the multidimensional selection coefficient $s=(\lambda, \delta, \alpha, \varepsilon)$, the probability $u$ is differentiable, and in a neighbourhood of $s=0$ (selective neutrality),

$$
u=p+v^{\prime} . s+o(s),
$$


where the selection gradient $v=\left(v^{\lambda}, v^{\delta}, v^{\alpha}, v^{\varepsilon}\right)$ can be expressed as

$$
\begin{aligned}
& v_{n, m}^{\iota}=p(1-p) g_{n+m}^{\iota}, \quad \iota \neq \varepsilon, \\
& v_{n, m}^{\varepsilon}=p(1-p)(1-2 p) g_{n+m}^{\varepsilon}
\end{aligned}
$$

where the $g$ 's depend solely on the resident's characteristics $b, c$, and on the total initial population size $n+m$. They are called the invasibility coefficients.

The invasibility coefficients of a pure resident population are interesting to study, as they provide insights about how the fixation probability deviates from $p$ as the selection coefficients of the mutant deviate from 0 . They provide information about the robustness of the resident population, i.e. its resistance to mutant invasions. In the simplest case where mutations in the parameter space are isotropic, the biggest invasibility coefficient gives the direction of the parameter space where a mutant is more likely to invade. More generally, when there are correlations between mutations in the parameter space, the likeliest direction of evolution in the trait space is given by the deterministic coefficient of the canonical diffusion (4), in which the fitness gradient is given by

$$
\nabla_{2} \chi(x, x)=a_{\lambda}(x) \nabla b(x)-a_{\delta}(x) \nabla_{1} c(x, x)+a_{\alpha}(x) \nabla_{2} c(x, x),
$$

where, for $\iota=\lambda, \delta, \alpha$,

$$
a_{\iota}(x)=e^{-\theta(x)} \sum_{n=1}^{\infty} \frac{n g_{n+1}^{\iota}(x) \theta(x)^{n-1}}{(n+1)^{2}(n-1) !} .
$$

It is possible to obtain explicit expressions for the invasibility coefficients $g^{\iota}$ as series. We refer to [2] for the exact expressions. In particular, these expressions yield that $a_{\iota}(x)=$ $\hat{a}_{\iota}(\theta(x)) / c(x, x)$ for some function $\hat{a}_{\iota}$. Moreover, they allow one to compute numerically the invasibility coefficients, and therefore the quantities $\hat{a}_{\iota}$ for $\iota=\lambda, \delta, \alpha$ as functions of the parameter $\theta(x)$. These numerical results can be used to make simulations of the canonical diffusion of adaptive dynamics in various ecological examples. In particular, in contrast with the classical canonical equation of adaptive dynamics, the presence of a genetic drift can induce the evolutionary dynamics to drift away from evolutionary stable strategies, where the fitness gradient is zero. When the fitness gradient admits several zeros, it can visit several basins of attraction on various timescales.

This numerical study is a work in progress, that is quite delicate because the series involved in the computation of $g^{\iota}$ are slowly converging, with first terms that grow exponentially fast with $\theta$. We shall give here some preliminary results. Fig. 1 shows the functions $\hat{a}_{\iota}$ for $\iota=\lambda, \delta, \alpha$. Several comments can be made from this figure. First, for any $\theta>0, \hat{a}_{\delta}(\theta)>\hat{a}_{\alpha}(\theta)>\hat{a}_{\lambda}(\theta)$. This means that, for equal mutation steps in the parameter space, a mutation is always more advantageous in the direction $\delta$ than in the direction $\alpha$, which is itself more advantageous than in the direction $\lambda$. In other words, in a given population, a better defence capacity is more beneficial than a harsher aggressiveness, which is more beneficial than a greater fertility.

Moreover, as $\theta$ goes to infinity, these functions have different asymptotic behaviours. $\hat{a}_{\delta}$ seems to converge to $1 / 2$, whereas $\hat{a}_{\lambda}(\theta)$ and $\hat{a}_{\alpha}(\theta)$ are both equivalent to $1 / 2 \theta$ (see Fig. 2). However, this does not mean that mutations are more likely to become fixed in the $\delta$ direction when $\theta$ is large, because the fact that $b=\theta c$ must be taken into account. 


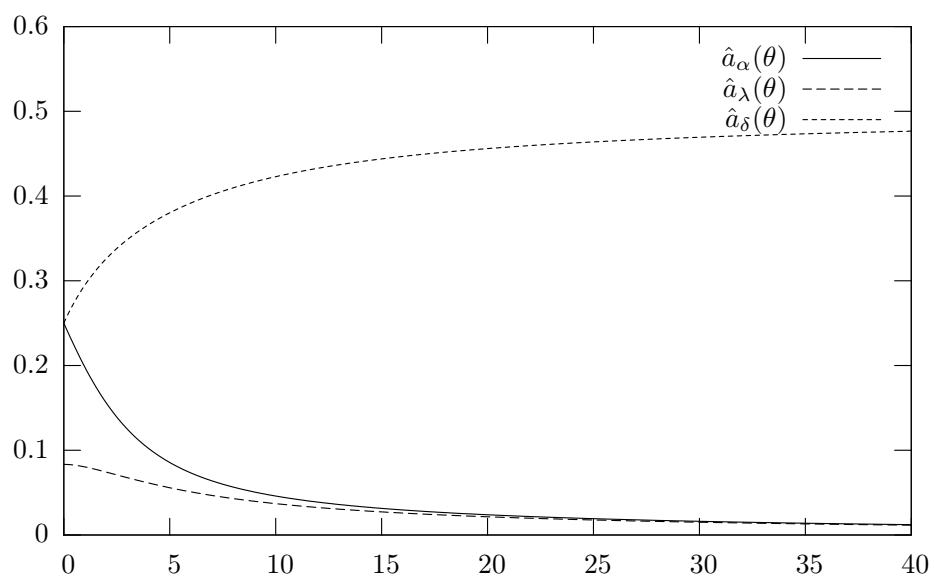

Fig. 1. The functions $\hat{a}_{\lambda}, \hat{a}_{\delta}$ and $\hat{a}_{\alpha}$ as functions of $\theta$

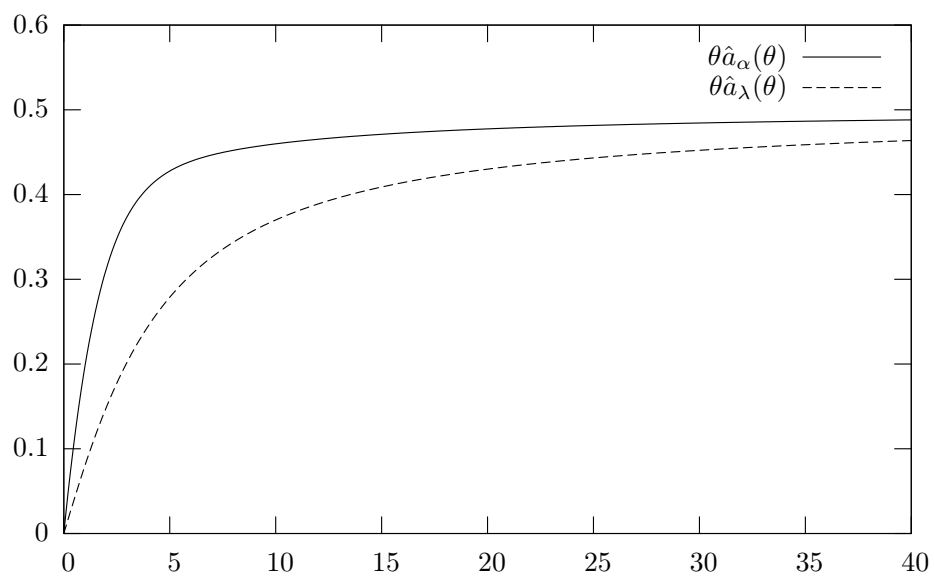

Fig. 2. The functions $\theta \mapsto \theta \hat{a}_{\lambda}(\theta)$ and $\theta \mapsto \theta \hat{a}_{\alpha}(\theta)$

The precise balance between the functions $a_{\iota}$ in $(7)$ when the population is large can be computed as follows: introduce a parameter $K$ in the microscopic model by dividing $c(\cdot, \cdot)$ by $K$ and consider the limit $K \rightarrow+\infty$ in the canonical diffusion. Denoting by $\chi_{K}(\cdot, \cdot)$ the fitness function obtained this way and using the asymptotic behaviours given above, one gets that

$$
\lim _{K \rightarrow+\infty} \nabla_{2} \chi_{K}(x, x)=\frac{1}{2 b(x)}\left(\nabla b(x)-\theta(x) \nabla_{1} c(x, x)\right) .
$$

Moreover, by $(5), \chi_{K}(x, x)$ converges to 0 when $K \rightarrow \infty$. Now, as proved in [1], the fitness function of the canonical equation of adaptive dynamics (1) is given by $f(x, y)=$ $b(y)-c(y, x) \theta(x)$. Therefore, as $K \rightarrow+\infty$, the canonical diffusion converges to a deterministic ODE which is precisely the canonical equation of adaptive dynamics. This gives a new justification of this equation, and allows one to study the fluctuations around the 
canonical equations when $K$ is large but finite. In particular, our diffusion equation with small diffusion term enters the framework of Freidlin-Wentzell's theory [4], which can be applied to predict the long time behaviour of the diffusion, and its chain of visit of basins of attractions when $K$ is large. This kind of information is biologically very relevant, since it allows one to predict in which order all the evolutionary stable strategies will be visited by the population and on which timescale.

\section{References}

[1] N. Champagnat, A microscopic interpretation for adaptive dynamics trait substitution sequence models, Stoch. Proc. Appl. 116 (2006), 1127-1160.

[2] N. Champagnat and A. Lambert, Evolution of discrete populations and the canonical diffusion of adaptive dynamics, Ann. Appl. Prob. 17 (2007), 102-155.

[3] U. Dieckmann and R. Law, The dynamical theory of coevolution: A derivation from stochastic ecological processes, J. Math. Biol. 34 (1996), 579-612.

[4] M. I. Freidlin and A. D. Wentzell, Random Perturbations of Dynamical Systems, SpringerVerlag, Berlin, 1984.

[5] T. F. Hansen, Stabilizing selection and the comparative analysis of adaptation, Evolution 51 (1997), 1341-1351.

[6] J. Hofbauer and R. Sigmund, Adaptive dynamics and evolutionary stability, Appl. Math. Letters 3 (1990), 75-79.

[7] A. Lambert, The branching process with logistic growth, Ann. Appl. Prob. 15 (2005), 1506-1535.

[8] R. Lande, Natural selection and random genetic drift in phenotypic evolution, Evolution 30 (1976), 314-334.

[9] J. A. J. Metz, R. M. Nisbet and S. A. H. Geritz, How should we define 'fitness' for general ecological scenarios?, Trends in Ecology and Evolution 7 (1992), 198-202.

[10] J. A. J. Metz, S. A. H. Geritz, G. Meszéna, F. A. J. Jacobs and J. S. van Heerwaarden, Adaptive Dynamics, a geometrical study of the consequences of nearly faithful reproduction, in: Stochastic and Spatial Structures of Dynamical Systems, S. J. van Strien and S. M. Verduyn Lunel (eds.), North-Holland, Amsterdam, 1996, 183-231. 